\title{
Multi-Channel Modulo Samplers Constructed from Gaussian Integers
}

\author{
Yicheng Gong, Lu Gan, Senior member, IEEE, and Hongqing Liu, Senior member, IEEE
}

\begin{abstract}
Recently, there is an increased interest in the study of modulo analog to digital converters (ADCs). These new systems can reconstruct a signal whose amplitude is much higher than the conventional ADC's dynamic range. Modulo ADCs are characterized by their modulo threshold and in the current literature, all existing works are limited to real-valued moduli. In this paper, we propose multi-channel modulo samplers with complex-valued moduli to sample a band-limited complex signal. Specifically, we discuss the construction of complex divisors from Gaussian integers and propose their efficient implementations. A memory-efficient, closed-form recovery algorithm is also proposed. Simulation results demonstrate that the proposed systems can provide stable reconstruction of a high dynamic range complex-valued signal at low sampling rates.
\end{abstract}

Index Terms-Analog-to-digital conversion (ADC), Complex signal representations, Modulo samplers, Chinese reminder theorem (CRT), Gaussian integers

\section{INTRODUCTION}

Complex signal representations have found many applications in radar, control and communications. In this paper, we consider the sampling of a continuous-time, finite-energy, band-limited complex-valued signal $g(t)=g_{I}(t)+i g_{Q}(t)$ with a maximum angular frequency of $\Omega$, where $g_{I}(t)$ and $g_{Q}(t)$ represent the real part (I-channel) and the imaginary part (Qchannel) of $g(t)$, respectively. We are interested in the scenario when the amplitudes of $g_{I}(t)$ or $g_{Q}(t)$ are much higher than the dynamic range of the analog-to-digital converter (ADC). Recently, unlimited sampling framework with modulo sampling hardware was developed in [1]-[4]. Specifically, for a given $x \in \mathbb{R}$ and $\Delta>0$, the modulo operation is defined as [1], [5]

$$
\langle x\rangle_{\Delta}=x \bmod \Delta \triangleq x-\Delta\left\lceil\frac{x}{\Delta}\right\rfloor,
$$

where $\lceil t\rfloor \triangleq \operatorname{argmin}_{b \in \mathbb{Z}}|t-b|$ is the "rounding" operation, which returns the closest integer to $t$ [1], [5].

According to Unlimited Sampling Theorem in [1], [3], $g(t)$ can be recovered from its modulo samples through higher order differences (HOD) reconstruction if the sampling period $T_{u s}$ satisfies $T_{u s} \leq 1 /(2 e \Omega)$. That is, for HOD, the required sampling rate $F_{s}$ is at least $2 e \pi f_{N Y Q} \approx 17.1 f_{N Y Q}$, in which $f_{N Y Q}$ is the Nyquist rate. For some wide-band applications, it

Yicheng Gong is with both Dept. of Electronic and Electrical Engineering, Brunel University, London, UK and International College, Chongqing University of Posts and Telecommunications, Chongqing, China. (Email: 1840657@brunel.ac.uk)

Lu Gan is with Dept. of Electronic and Electrical Engineering, Brunel University, London, UK. (Email: lu.gan@brunel.ac.uk)

Hongqing Liu is with School of Communications and Information Engineering, Chongqing University of Posts and Telecommunications, Chongqing, China. (Email: hongqingliu @ cqupt.edu.cn)
TABLE I: Comparison of different modulo samplers for noisefree reconstruction

\begin{tabular}{|c|c|c|c|c|}
\hline Methods & $L$ & Minimum $F_{s}$ & $g_{\max }$ & Same $\left|\Delta_{l}\right|$ \\
\hline$[1],[3]$ & 1 & $2 \pi e f_{N Y Q}$ & $<\infty$ & Yes \\
\hline$[5]$ & 1 & $>f_{N Y Q}$ & $<\infty$ & Yes \\
\hline$[13]$ & 2 & $2 f_{N Y Q}$ & $<\tau_{0} \Delta / 2$ & No \\
\hline This work & 2 & $2 f_{N Y Q}$ & $<\left|\tau_{0}\right| \Delta / 2$ & Yes \\
\hline
\end{tabular}

${ }^{1}$ Restriction: There exists a negative integer $N$ so that $|g(n / F s)|<\Delta / 2$ when $n<N$ [5].

may be difficult to achieve such a high sampling rate. In [5], it was shown that if $F_{s}>f_{N Y Q}$, reconstruction is possible by using a prediction filter constructed from Chebyshev polynomials $(\mathrm{ChP})$. However, this method requires the knowledge of unfolded samples, i.e., there exists a negative integer $N$ so that $|g(n / F s)|<\Delta / 2$ when $n<N$. For real-world applications, this condition is often impractical. Additionally, this method is sensitive to noises. For other works on single-channel modulo ADCs, we refer the readers to [2], [6]-[12] and the references therein.

In [13], multi-channel modulo samplers were developed based on Chinese remainder theorem (CRT). In an $L$-channel modulo ADC, each channel samples at $f_{N Y Q}$ with different dynamic ranges $\Delta_{0}<\Delta_{1}<\cdots<\Delta_{L-1}$

$$
\Delta_{l}=\epsilon \tau_{l} \quad 0 \leq l \leq L-1,
$$

where $\epsilon$ is a positive real number and $\tau_{l}(0 \leq l \leq L-1)$ are $L$ jointly prime positive integers. In the noiseless case, only 2-channels are required with a total sampling rate of $F_{s}=2 f_{N Y Q}$. Besides, each signal sample can be recovered independently [13]. However, once $\Delta_{l}$ are fixed, the maximum signal amplitude $g_{\max }$ for reconstruction is bounded by $P \epsilon / 2$, in which $P$ is the least common multiple $(\mathrm{lm})$ of $\tau_{l}$. In addition, as $\Delta_{l}$ are different, the calibrations of ADC dynamic ranges may be complicated.

In this paper, we propose multi-channel modulo samplers with complex-valued moduli $\Delta_{l}$. Specifically, the positive integers $\tau_{l}$ in (2) are generalized to Gaussian integers of the form $\tau_{l}=p_{l}+i q_{l}$, in which $p_{l}$ and $q_{l}$ are non-negative integers. Our main contributions include: $i$ ) Selection of moduli: We propose to use pairwise co-prime Gaussian integers $\tau_{l}$ in (2), which allows for recovery of a signal with larger dynamic range than that of real-valued moduli (Section II). We prove that perfect recovery can be achieved at a total sampling rate of $2 f_{N Y Q}$ (Proposition 1). ii) Implementation: We develop implementation diagram of complex modulo operations by applying analog phase-shifts before modulo ADCs (Section III-A). In the special case when $L=2$, all modulo 


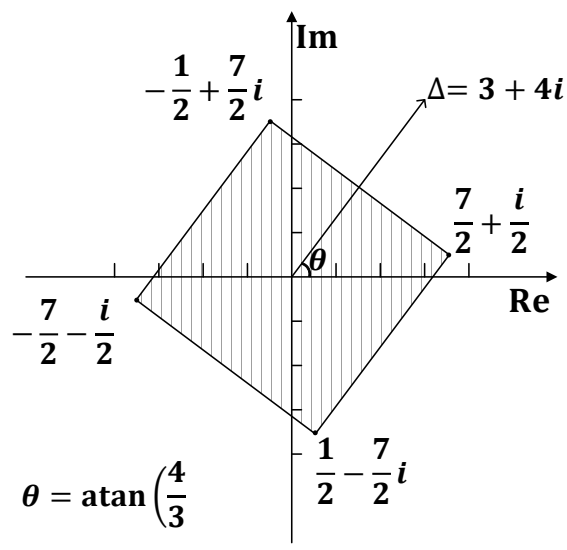

Fig. 1: Region of complex remainder sets $\mathbb{S}(3+4 i)$.

ADCs have the same dynamic range in our proposed systems, which is impossible for multi-channel systems developed in [13]. iii) Reconstruction: For two co-prime Gaussian integers $x_{0}=p+i q$ and $x_{1}=p-i q$, we prove that $x_{0}^{-1} \bmod x_{1}$ is a rational integer (Proposition 2). Based on robust CRT of [14], a 2-stage memory-efficient reconstruction algorithm can be obtained. Simulation results in Section IV demonstrate the effectiveness of the proposed systems. As a quick summary, Table I lists the required total sampling rate $F_{s}$ and maximum achievable signal amplitude $g_{\max }$ for different modulo samplers in noiseless measurements.

Notations: Throughout this paper, $i=\sqrt{-1}$ and $e=$ $\exp (1)$. The set of rational integers is denoted as $\mathbb{Z}$. For a real number $x,\lceil x\rceil$ and $\lceil x\rfloor$ correspond to the ceiling and rounding operations, respectively. For a complex number $x$, $\operatorname{Re}(x)$ and $\operatorname{Im}(x)$ are its real and imaginary parts, respectively. The rounding operation is given by $\lceil x\rfloor=\lceil\operatorname{Re}(x)\rfloor+i\lceil\operatorname{Im}(x)\rfloor$. For a non-zero complex modulus $\Delta,\langle x\rangle_{\Delta}$ represents the modulo operation in (1). The fundamental domain of $\Delta$ is denoted as $\mathbb{S}(\Delta)$, which represents its remainder set [15]:

$$
\mathbb{S}(\Delta)=\left\{\Delta(\alpha+i \beta),-\frac{1}{2}<\alpha, \beta \leq \frac{1}{2}\right\} .
$$

Geometrically, $\mathbb{S}(\Delta)$ is a rotated square with 4 vertices at $\frac{\Delta}{2}(1+i), \frac{\Delta}{2}(1-i), \frac{\Delta}{2}(-1-i)$ and $\frac{\Delta}{2}(-1+i)$. As an example, Fig. 1 shows $\mathbb{S}(3+4 i)$. For a complex signal $g(t)=g_{I}(t)+i g_{Q}(t), g_{\max }$ is given by

$$
g_{\max }=\max \left(\left\|g_{I}(t)\right\|_{\infty},\left\|g_{Q}(t)\right\|_{\infty}\right),
$$

where the subscript $\infty$ denotes the infinity norm.

\section{Selection of Complex moduli}

In this section, we first introduce basics of Gaussian integers and then present muli-channel modulo samplers constructed from complex moduli. A Gaussian integer $x$ can be written as $x=p+i q$ with $p, q \in \mathbb{Z}$. Its norm $\mathbb{N}(x)$ is given by [16]

$$
\mathbb{N}(x)=x \bar{x}=|x|^{2}=p^{2}+q^{2},
$$

where $\bar{x}$ is the complex conjugate of $x$. For two Gaussian integers $x_{0}$ and $x_{1}$, they are relatively prime (or co-prime) if and only if there exist two Gaussian integers $\alpha$ and $\beta$ so that the Bézout's identity holds, i.e., $x_{0} \alpha+x_{1} \beta=1$. Alternatively,
TABLE II: Comparisons of $g_{\max }$ for real- and complex-valued moduli for $\left|\Delta_{l}\right| \leq 5$ and $\epsilon=1$

\begin{tabular}{|c|c|c|c|}
\hline$L$ & Moduli & $\Delta_{l}$ & $g_{\max }$ \\
\hline \multirow{2}{*}{2} & Real [13] & 5,4 & $<10$ \\
\cline { 2 - 4 } & Complex & $3+4 i, 3-4 i$ & $<12.5$ \\
\hline \multirow{2}{*}{3} & Real [13] & $5,4,3$ & $<30$ \\
\cline { 2 - 4 } & Complex & $3+4 i, 3-4 i, 4$ & $<50$ \\
\hline \multirow{2}{*}{4} & Real [13] & $5,4,3,1$ & $<30$ \\
\cline { 2 - 4 } & Complex & $3+4 i, 3-4 i, 1+4 i, 1-4 i$ & $<212.5$ \\
\hline
\end{tabular}

$x_{0}=p_{0}+i q_{0}$ and $x_{1}=p_{1}+i q_{1}$ are co-prime if and only if their greatest common divisor (gcd) satisfy [17]

$$
\operatorname{gcd}\left(\mathbb{N}\left(x_{0}\right), \mathbb{N}\left(x_{1}\right), p_{0} p_{1}+q_{0} q_{1}\right)=1 .
$$

In the special case when $x_{0}=p+i q$ and $x_{1}=p-i q$, (6) can be simplified as [18]

$$
\operatorname{gcd}(p, q)=1 \quad \text { and } \quad|p-q| \bmod 2=1 .
$$

That is, $p, q$ are co-prime with different parities, i.e., one odd and one even.

Next, we propose a multi-channel modulo sampler constructed from $L$ complex divisors $\Delta_{l}(0 \leq l \leq L-1)$, where $\tau_{l}$ in (2) are pairwise co-prime Gaussian integers. Besides, in the first $2 L_{1}$ divisors, $\tau_{2 l}$ and $\tau_{2 l+1}\left(0 \leq l \leq L_{1}-1\right)$ are complex conjugate pairs:

$$
\tau_{2 l}=p_{l}+i q_{l} \quad \text { and } \quad \tau_{2 l+1}=p_{l}-i q_{l},
$$

where $p_{l}$ and $q_{l}$ are positive integers. For the remaining $L-$ $2 L_{1}$ divisors, $\tau_{l^{\prime}}\left(l^{\prime}=2 L_{1}, \cdots, L-1\right)$ are positive integers. According to the generalized CRT [14], a complex number $x$ can be perfectly recovered from $L$ remainders $\langle x\rangle_{\Delta_{l}}(l=$ $0, \cdots, L-1)$ if $x \in \mathbb{S}(P \epsilon)$, or equivalently,

$$
|\operatorname{Re}(x)|<P \epsilon / 2 \quad \text { and }|\operatorname{Im}(x)|<P \epsilon / 2,
$$

where $P=\prod_{l=0}^{L_{1}-1}\left(p_{l}^{2}+q_{l}^{2}\right) \cdot \prod_{l^{\prime}=2 L_{1}}^{L-1} \tau_{l^{\prime}}$.

Hereafter, we will assume that $\left|\Delta_{l}\right| \leq \Delta_{\max }(l=$ $0,1, \cdots, L-1)$, i.e., their magnitudes are bounded by $\Delta_{\max }$. The following example shows that our proposed system can offer a larger signal dynamic range than that of the multichannel system with only real-valued moduli [13].

Example 1: In this example, we compare the upper bound of $g_{\max }$ for complex and real moduli [13]. Suppose that $\epsilon=1$ and we restrict $\left|\Delta_{l}\right| \leq 5$. For $L=2,3$ and 4, Table II lists the corresponding $\Delta_{l}(l=0, \cdots, L-1)$ that can achieve maximum $g_{\max }$. This table implies that by extending $\tau_{l}$ to Gaussian integers, one can sample a signal with a much higher dynamic range.

The next proposition proves that with a careful selection of $\tau_{l}$ and $\epsilon$, a band-limited signal $g(t)$ with known $g_{\max }$ can be recovered at $F_{s}=2 f_{N Y Q}$ and $\left|\Delta_{0}\right|=\left|\Delta_{1}\right|=\Delta_{\max }$.

Proposition 1: Suppose that $g(t)$ is a finite-energy, bandlimited complex-valued signal with $g_{\max }$ defined as in (4). Assume that the maximum dynamic range of modulo ADC is $\Delta_{\max }$. Consider a 2-channel modulo sampler with $\Delta_{l}=\tau_{l} \epsilon$ $(l=0,1)$ and $\tau_{0}=p+i q$ and $\tau_{1}=p-i q$, in which

$$
p=\left\lceil\frac{2 g_{\max }}{\Delta}\right\rceil, \quad q=\left\{\begin{array}{cc}
1 ; & p \text { even } \\
2 ; & p \text { odd }
\end{array}, \quad \epsilon=\frac{\Delta_{\max }}{\sqrt{p^{2}+q^{2}}} .\right.
$$




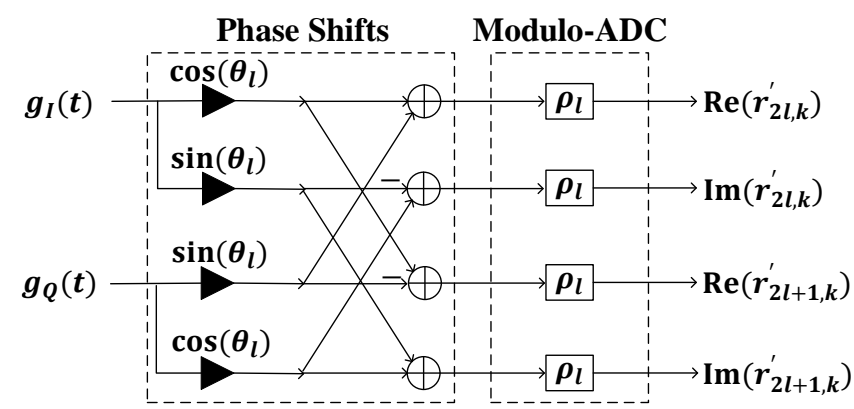

Fig. 2: Block diagram to generate $r_{2 l, k}^{\prime}=\left\langle g_{k} e^{-j \theta_{l}}\right\rangle_{\rho_{l}}+\delta_{2 l, k}^{\prime}$ and $r_{2 l+1, k}^{\prime}=\left\langle g_{k} e^{j \theta_{l}}\right\rangle_{\rho_{l}}+\delta_{2 l+1, k}^{\prime}$.

Then, $g(t)$ can be completely recovered from noise-free modulo samples $\left\langle g_{k}\right\rangle_{\Delta_{l}}(l=0,1)$ with $g_{k}=g\left(k / f_{N Y Q}\right)(k \in \mathbb{Z})$.

Proof: From (10), it is clear that $\operatorname{gcd}(p, q)=1$ and $p, q$ have different parities. Thus, $\tau_{0}$ and $\tau_{1}$ are co-prime. Besides, it is straightforward $\left|\Delta_{0}\right|=\left|\Delta_{1}\right|=\Delta_{\max }$. By (9)-(10), the maximum achievable range is $\left|\tau_{0}\right| \Delta_{\max } / 2=$ $\sqrt{p^{2}+q^{2}} \Delta_{\max } / 2>p \Delta_{\max } / 2 \geq g_{\max }$. Thus, if each channel is sampled at the Nyquist rate $f_{N Y Q}, g_{k}=g\left(k / f_{N Y Q}\right)$ can be reconstructed perfectly from $\left\langle g_{k}\right\rangle_{\Delta_{l}}(l=0,1)$. That is, the total sampling rate is $F_{s}=2 f_{N Y Q}$.

Remarks: $i$ ) The selection of $p$ and $q$ in (10) is not unique. ii) Recall that the minimum $F_{s}$ is also $2 f_{N Y Q}$ for multi-channel system with real-valued moduli [13]. However, the modulo ADCs in [13] need to have different dynamic ranges, which makes it difficult for ADC calibration.

\section{IMPLEMENTATION AND RECONSTRUCTION}

\section{A. Implementation}

The previous subsection shows that with complex divisors, one can reconstruct a signal with higher dynamic range. Note that the dynamic range of a modulo ADC is a positive real number. One may wonder how to implement modulo operations for complex divisors? To address this issue, we first express $\Delta_{2 l}$ and $\Delta_{2 l+1}\left(0 \leq L_{1}-1\right)$ in polar forms

$$
\Delta_{2 l}=\rho_{l} e^{j \theta_{l}}, \quad \Delta_{2 l+1}=\rho_{l} e^{-j \theta_{l}},
$$

in which $\rho_{l}=\epsilon \sqrt{p_{l}^{2}+q_{l}^{2}}$ and $\theta_{l}=\arctan \left(q_{l} / p_{l}\right)$. Denote $g_{k}=g\left(k / f_{N Y Q}\right)$ and define $y_{2 l, k}^{\prime}$ and $y_{2 l, k}^{\prime}$ as follows

$$
y_{2 l, k}^{\prime}=\left\langle g_{k} e^{-j \theta_{l}}\right\rangle_{\rho_{l}}, \quad y_{2 l+1, k}^{\prime}=\left\langle g_{k} e^{j \theta_{l}}\right\rangle_{\rho_{l}} .
$$

From (11) and (12), one can easily prove

$$
y_{2 l, k}^{\prime} e^{j \theta_{l}}=\left\langle g_{k}\right\rangle_{\Delta_{2 l}}, \quad y_{2 l+1, k}^{\prime} e^{-j \theta_{l}}=\left\langle g_{k}\right\rangle_{\Delta_{2 l+1}} .
$$

Note that $y_{2 l, k}^{\prime}$ and $y_{2 l+1, k}^{\prime}$ in (12) can be acquired by

1) Applying phase shifts to $g(t)$ to produce $g_{2 l}(t)=$ $g(t) e^{-j \theta_{l}}$ and $g_{2 l+1}(t)=g(t) e^{j \theta_{l}}$;

2) Sampling $g_{2 l}(t)$ and $g_{2 l+1}(t)$ using modulo ADCs with dynamic range $\rho_{l}$.

Phase shifting of $g(t)$ by $-\theta_{l}$ and $\theta_{l}$ are equivalent to multiplying $\left[\begin{array}{ll}g_{I}(t) & g_{Q}(t)\end{array}\right]^{T}$ with $\mathbf{R}_{l}$ and $\hat{\mathbf{R}}_{l}$, respectively

$$
\mathbf{R}_{l}=\left[\begin{array}{cc}
\cos \left(\theta_{l}\right) & \sin \left(\theta_{l}\right) \\
-\sin \left(\theta_{l}\right) & \cos \left(\theta_{l}\right)
\end{array}\right], \quad \hat{\mathbf{R}}_{l}=\left[\begin{array}{cc}
\cos \left(\theta_{l}\right) & -\sin \left(\theta_{l}\right) \\
\sin \left(\theta_{l}\right) & \cos \left(\theta_{l}\right)
\end{array}\right] .
$$

The corresponding implementation diagram is shown in Fig. 2. Here, the outputs $r_{2 l, k}^{\prime}$ and $r_{2 l+1, k}^{\prime}$ are given by

$$
r_{2 l, k}^{\prime}=y_{2 l, k}^{\prime}+\delta_{2 l, k}^{\prime}, \quad r_{2 l+1, k}^{\prime}=y_{2 l+1, k}^{\prime}+\delta_{2 l+1, k}^{\prime}
$$

in which $\delta_{2 l, k}^{\prime}$ and $\delta_{2 l+1, k}^{\prime}$ are errors introduced by modulo ADCs. Eq. (13) suggests we can use $r_{2 l, k}^{\prime} e^{j \theta}$ and $r_{2 l, k}^{\prime} e^{-j \theta}$ to approximate modulo samples $\left\langle g_{k}\right\rangle_{\Delta_{2 l}}$ and $\left\langle g_{k}\right\rangle_{\Delta_{2 l+1}}$ for reconstruction.

Note that in the special case with $L=2$, Fig. 2 indicates that 4 modulo ADCs with the same dynamic range $\rho_{0}$ are required. If $\tau_{0}$ and $\tau_{1}$ are positive integers as proposed in [13], 4 modulo ADCs with 2 different dynamic ranges are needed. Thus, the proposed systems offer simpler ADC calibrations. The extra cost here is the analog phase rotations.

\section{B. Reconstruction}

We now consider the reconstruction of $g_{k}$ from noisy modulo samples. Since CRT is applicable to Gaussian integers, one can use any existing recovery algorithms by replacing positive integers with Gaussian ones [14], [19]-[28]. We consider robust CRT (RCRT) [14] as it has a closed-form solution with fast implementation. Just as classical CRT, RCRT requires precalculated modular multiplicative inverses. Simply speaking, for two co-prime Gaussian integers $a$ and $b$, a Gaussian integer $c \equiv a^{-1} \bmod b$ is $a$ 's modular multiplicative inverse modulo $b$ if $c a \bmod b=1$. For general Gaussian integers $a$ and $b, c$ is complex-valued. But for co-prime pairs $x_{0}=p+i q$ and $x_{1}=p-i q$, Proposition 2 shows that $x_{0}^{-1} \bmod x_{1}$ is an integer in $\mathbb{Z}$.

Proposition 2: Suppose that $x_{0}=p+i q$ and $x_{1}=p-i q$ are co-prime with $p, q \in \mathbb{Z}^{+}$. Then, the modular multiplicative inverse $c \equiv x_{0}^{-1} \bmod x_{1}$ is a rational integer.

Proof: By (7), the co-prime condition of $x_{0}$ and $x_{1}$ implies $\operatorname{gcd}(p, q)=1$ and $p, q$ have different parities. Without loss of generality, we assume that $p$ is odd and $q$ is even. Under such an assumption, it is clear that $p^{2}+q^{2}$ is an odd number, i.e., $\operatorname{gcd}\left(p^{2}+q^{2}, 2\right)=1$. Besides, as $\operatorname{gcd}(p, q)=1$, $\operatorname{gcd}\left(p^{2}+q^{2}, p\right)=1$. Therefore, $\operatorname{gcd}\left(p^{2}+q^{2}, 2 p\right)=1$. By Bézout's identity, there exist two integers $c, d \in \mathbb{Z}$ so that

$$
c \cdot 2 p+d \cdot\left(p^{2}+q^{2}\right)=1
$$

As $2 p=x_{0}+x_{1}$ and $p^{2}+q^{2}=x_{0} x_{1}$, we have

$$
c x_{0}+c x_{1}+d x_{0} x_{1}=1,
$$

which implies that $c x_{0} \bmod x_{1}=1$, i.e., $c$ is the multiplicative inverse of $x_{0}$ modulo $x_{1}$. When $q$ is odd and $p$ is even, the proof can be done in a similar way.

Based on the above proposition, we propose a 2-stage reconstruction algorithm as in [21]. In the first stage, RCRT [14] is applied to erroneous remainders of $\Delta_{2 l}$ and $\Delta_{2 l+1}$ for $l=0,1, \cdots, L_{1}-1$ to solve $\hat{x}_{l, k}$

$$
\begin{aligned}
\left\langle\hat{x}_{l, k}\right\rangle_{\Delta_{2 l}} & \approx r_{2 l, k}^{\prime} e^{j \theta_{l}}, \\
\left\langle\hat{x}_{l, k}\right\rangle_{\Delta_{2 l+1}} & \approx r_{2 l+1, k}^{\prime} e^{-j \theta_{l}} .
\end{aligned}
$$

By Proposition 2, the modular multiplicative inverses $\tau_{2 l}^{-1} \bmod \tau_{2 l+1}$ are integers in $\mathbb{Z}$. In the second stage, we aim 
to get reconstructed signal sample $\hat{g}_{k}$ through RCRT [14]

$$
\begin{aligned}
\left\langle\hat{g}_{k}\right\rangle_{N_{l} \epsilon} & \approx \hat{x}_{l, k}, \quad l=0, \cdots, L_{1}-1 \\
\left\langle\hat{g}_{k}\right\rangle_{\Delta_{l}^{\prime}} & \approx r_{l^{\prime}, k}, \quad l^{\prime}=2 L_{1}, \cdots, L-1
\end{aligned}
$$

in which $N_{l}=p_{l}^{2}+q_{l}^{2}$ and $r_{l^{\prime} k}=\left\langle g_{k}\right\rangle_{\Delta_{l^{\prime}}}+\delta_{l^{\prime}, k}$ with $\delta_{l^{\prime}, k}$ being the ADC error $\left(l^{\prime}=2 L_{1}, \cdots L-1\right)$. As $N_{l}$ and $\tau_{l}^{\prime}$ are positive integers, all the modular multiplicative inverses are positive integers. Thus, the proposed algorithm is memory efficient as we only need to store rational integers. In addition, following a similar analysis as in [14], [21], one can show that if the errors from modulo samplers satisfy

$$
\left|\delta_{l, k}^{\prime}\right|<\epsilon / 4, \quad\left|\delta_{l^{\prime}, k}\right|<\epsilon / 4,
$$

stable reconstruction can be achieved with $\left|\hat{g}_{k}-g_{k}\right|<\epsilon / 4$.

Remark: For $L=2$, Eq. (10) implies that $\epsilon$ becomes small when $g_{\max }$ gets large. To improve the system's robustness to ADC noises, over-sampling is required by increasing $\epsilon$ and $L$ under the constraint $\left|\Delta_{l}\right| \leq \Delta_{\max }$.

\section{Simulations}

We report simulation results for different modulo ADCs to sample complex-valued, band-limited signals $g(t)$ with maximum angular frequency $\Omega=\pi \mathrm{rad} / \mathrm{s}\left(f_{N Y Q}=1 \mathrm{~Hz}\right)$. We restrict $\left|\Delta_{l}\right| \leq 5$ in all simulations. For multi-channel systems, $\epsilon=1$ and each channel is sampled at $1 \mathrm{~Hz}$. We run simulations with $L=2\left(F_{s}=2 \mathrm{~Hz}\right)$ and $L=3\left(F_{s}=3\right.$ $\mathrm{Hz}$ ) for both real-valued [13] and complex-valued moduli. The corresponding values of $\Delta_{l}$ and achievable $g_{\max }$ can be found in Table II. For single-channel systems, we fix $\Delta=5$ and consider $i$ ) HOD reconstruction [1], [3] at $F_{s}=18 \mathrm{~Hz}$ and ii) ChP-based reconstruction [5] for $F_{s}=2 \mathrm{~Hz}$ and $F_{s}=3 \mathrm{~Hz}$, respectively. Each test signal $g(t)$ can be expressed as

$$
g(t)=\sum_{k=-10}^{10} A \cdot\left(a_{k}+i b_{k}\right) \operatorname{sinc}(t-k), \quad-40 \leq t<40
$$

where $\operatorname{sinc}(t)=\frac{\sin (\pi t)}{\pi t}$ is an ideal low-pass filter in $[-\pi, \pi]$. Coefficients $a_{k}$ and $b_{k}$ are uniformly distributed in $[-1,1]$ and $A$ is a constant to obtain different $g_{\max }$. The input signal to noise ratio (SNR) follows the same definition as that in [7] and varies from $10 \mathrm{~dB}$ to $40 \mathrm{~dB}$ with a step size of $2 \mathrm{~dB}$. For each input SNR, we produce $Q=10^{4}$ realizations of $g(t)$. The root relative square error (RRSE) is used to quantify reconstruction error:

$$
\operatorname{RRSE}=\sqrt{\frac{\sum_{n}\left|g\left(n / f_{s}\right)-\hat{g}\left(n / f_{s}\right)\right|^{2}}{\sum_{n}\left|g\left(n / f_{s}\right)\right|^{2}}},
$$

where $f_{s}=F_{s} / L$ is the sampling frequency for each channel.

Fig. 3a and Fig. 3b show the results of average RRSE for $g_{\max }=9.5$ and $g_{\max }=48$, respectively. In these figures, $\kappa$ denotes the order of finite difference in HOD-based reconstruction [1], [3]. For ChP-based method [5], the filter order is $2 K$, where $K$ represents the order of Chebychev polynomial calculated from Eq. (9) in [5]. One can observe that for $g_{\max }=9.5$, HOD [1], [3] offers the best performance at $F_{s}=18 \mathrm{~Hz}$ for input SNR from 12 to $20 \mathrm{~dB}$. In fact, HOD reconstruction can work at sampling rates lower than

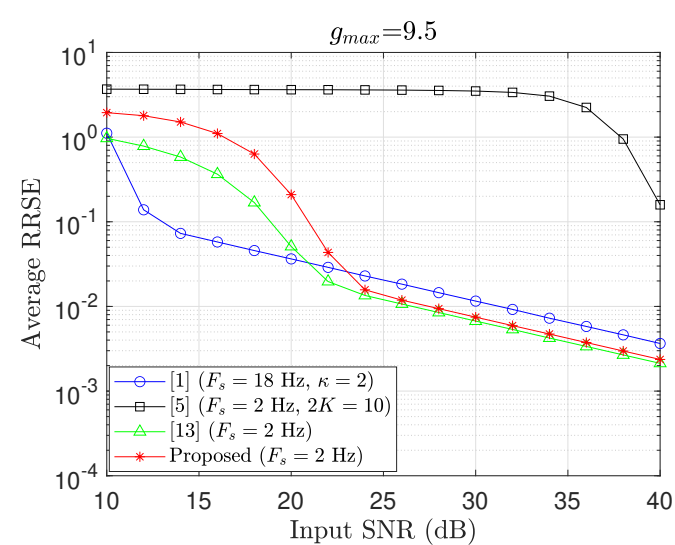

(a)

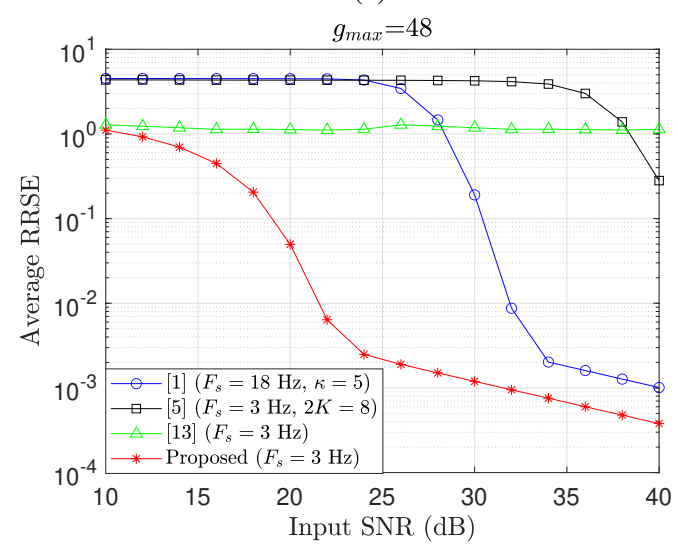

(b)

Fig. 3: Reconstruction results for different modulo samplers (a) $g_{\max }=9.5$ (b) $g_{\max }=48$.

$2 e \pi f_{N Y Q}$ for high SNR [3]. However, HOD's performance degrades for $g_{\max }=48$ and low SNR. For ChP-based reconstruction [5], it produces large reconstruction error at $F_{s}=2 \mathrm{~Hz}$ or $F_{s}=3 \mathrm{~Hz}$ even at high SNR. For multi-channel systems in [13], they work well for $g_{\max }=9.5$, but fail at $g_{\max }=48$ as the signal's amplitude exceeds the maximum achievable dynamic range. Our proposed systems offer the best performance at $g_{\max }=48$ and they are slightly worse than the system in [13] for $g_{\max }=9.5$. Thus, they hold great potential to sample a high dynamic range complex-valued signal at low sampling rates.

\section{CONCLUSIONS}

This paper proposes multi-channel modulo sampling systems based on Gaussian integers. In particular, we discuss the selection of complex divisors and propose implementations through modulo ADC systems. Besides, a memory efficient, closed-form reconstruction algorithm is presented for robust recovery. Compared with multi-channel modulo samplers with only real-valued divisors [13], the proposed systems can recover a signal with higher dynamic range. Compared with single-channel modulo samplers [3], [5], our system can offer stable reconstruction with a provable error bound at much lower sampling rates. 


\section{REFERENCES}

[1] A. Bhandari, F. Krahmer, and R. Raskar, "On unlimited sampling," in 2017 International Conferences on Sampling Theory and Applications (SampTA), Tallinn, Estonia, Jul. 2017, pp. 31-35.

[2] — - "Unlimited sampling of sparse signals," in IEEE Intl. Conference on Acoustics, Speech and Signal Processing (ICASSP, Calgary, Canada, Apr. 2018, pp. 4569-4573.

[3] A. Bhandari, F. Krahmer, and R. Raskar, "On unlimited sampling and reconstruction," IEEE Transactions on Signal Processing, pp. 1-1, 2020, Early access, available at https://ieeexplore.iee.org/document/9282196.

[4] A. Bhandari, F. Krahmer, and T. Poskitt. (2021) Unlimited sampling from theory to practice: Fourier-Prony recovery and prototype ADC. [Online]. Available: https://arxiv.org/abs/2105.05818

[5] E. Romanov and O. Ordentlich, "Above the Nyquist rate modulo folding does not hurt," IEEE Signal Process. Lett., vol. 26, pp. 1167-1171, Aug. 2019.

[6] A. Bhandari, F. Krahmer, and R. Raskar, "Unlimited sampling of sparse sinusoidal mixtures," in IEEE Intl. Conference on Information theory (ISIT), Colorado, USA, Jun. 2018, pp. 336-340.

[7] S. Rudresh, A. Adiga, B. A. Shenoy, and C. S. Seelamantula, "Waveletbased reconstruction for unlimited sampling," in IEEE Intl. Conference on Acoustics, Speech and Signal Processing (ICASSP), Calgary, Canada, Apr. 2018, pp. 4584-4588.

[8] M. Cucuringu and H. Tyagi, "On denoising modulo 1 samples of a function," in the 21st Intl. Conference on Artificial Intelligence and Statistics, Canary Islands, Apr. 2018, pp. 1868-1876.

[9] O. Musa, P. Jung, and N. Goert, "Generalized approximate message passing for unlimited sampling of sparse signals," in in IEEE Global Conf. on Signal and Information Proc. (GlobalSIP),, Ahaheim, California, USA, Nov. 2018, pp. 336-340.

[10] V. Shah and C. Hegde, "Signal reconstruction from modulo observations," in 2019 IEEE Global Conference on Signal and Information Processing (GlobalSIP), 2019, pp. 1-5.

[11] V. Bouis, F. Krahmer, and A. Bhandari, "Multidimensional unlimited sampling: A geometrical perspective," in 2020 28th European Signal Processing Conference (EUSIPCO), Amsterdam, Netherlands, 2020, pp. 2314-2318.

[12] S. Fernández-Menduiña, F. Krahmer, G. Leus, and A. Bhandari, "DoA estimation via unlimited sensing," in 2020 28th European Signal Processing Conference (EUSIPCO), Amsterdam, Netherlands, 2021, pp. 1866-1870.

[13] L. Gan and H. Liu, "High dynamic range sensing using multi-channel modulo samplers," in 2020 IEEE 11th Sensor Array and Multichannel Signal Processing Workshop (SAM), Hangzhou, China, 2020, pp. 1-5.

[14] W. Wang and X.-G. Xia, "A closed-form robust Chinese remainder theorem and its performance analysis," IEEE Trans. Signal Process., vol. 58 , pp. 5655-5666, Nov. 2010.

[15] D. Munuera-Merayo. (2021) On Mignotte secret sharing schemes over gaussian integers. [Online]. Available: https://arxiv.org/abs/2104.06361

[16] K. Conrad, "The Gaussian Integers," Available at https://kconrad.math. uconn.edu/math5230f12/handouts/Zinotes.pdf.

[17] C. Li, L. Gan, and C. Ling, "Coprime sensing via Chinese remaindering over quadratic fields-Part II: Generalizations and applications," IEEE Transactions on Signal Processing, vol. 67, no. 11, pp. 2911-2922, 2019.

[18] _ - "Coprime sensing via Chinese remaindering over quadratic fieldsPart I: Array designs," IEEE Transactions on Signal Processing, vol. 67, no. 11, pp. 2898-2910, 2019

[19] X. P. Li, X.-G. Xia, W. J. Wang, and W. Wan, "A robust generalized Chinese remainder theorem for two integers," IEEE Trans. Inf. Theory, vol. 65, pp. 7491-7504, Dec. 2016.

[20] W. Wang, X. Li, W. Wang, and X.-G. Xia, "Maximum likelihood estimation based robust Chinese remainder theorem for real numbers and its fast algorithm," IEEE Trans. Signal Process., vol. 63, pp. 3317-3331, Jul. 2015.

[21] L. Xiao, X.-G. Xia, and W. J. Wang, "Multi-stage robust Chinese remainder theorem," IEEE Trans. Signal Process., vol. 62, pp. 4772-4785, Sep. 2014.

[22] L. Xiao, X.-G. Xia, and X.-G. Xia, "Towards robustness in residue number systems," IEEE Trans. Signal Process., vol. 65, pp. 1497-1510, Mar. 2017.

[23] A. Akhlaq, R. G. McKilliam, and R. Subramanian, "Basis construction for range estimation by phase unwrapping," IEEE Signal Process. Lett., vol. 22, pp. $2152-2156$, Nov. 2015

[24] A. Akhlaq, R. G. McKilliam, R. Subramanian, and A. Pollok, "Selecting wavelengths for least squares range estimation," IEEE Trans. Signal Process., vol. 64, pp. 5205 - 5216, Oct. 2016.
[25] W.-C. Li, X.-Z. Wang, and B. Moran, "Distance estimation using wrapped phase measurements in noise," IEEE Trans. Signal Process., vol. 61, pp. 1676-1688, Apr. 2013.

[26] _ "Wireless signal travel distance estimation using non-coprime wavelengths," IEEE Signal Process. Lett., vol. 24, pp. 27-31, Jan. 2017.

[27] L. Xiao and X.-G. Xia, "Frequency determination from truly subnyquist samplers based on robust Chinese remainder theorem," Signal Processing, vol. 150, pp. 248-258, Sep. 2018.

[28] L. Xiao, X. G. Xia, and Y. P. Wang, "Exact and robust reconstructions of integer vectors based on multidimensional Chinese Remainder Theorem (MD-CRT)," IEEE Transactions on Signal Processing, vol. 68, pp. $5349-5364,2020$ 